\title{
Cognición y estilo comunicativo: el sujeto posverbal y el objeto sintáctico*
}

\author{
Cognition and communicative style: postverbal subject and syntactic object
}

\begin{abstract}
María José Serrano
Universidad de La Laguna, Departamento de Filología Española, Tenerife, España. Correo electrónico: mjserran@ull.edu.es
\end{abstract}

En este trabajo se atribuyen distintos grados de prominencia y de informatividad textual a las tres variantes posicionales posibles del sujeto posverbal y del objeto verbal expreso (VSO, VOS y OVS) desde un punto de vista cognitivo para determinar el significado que con ellas se crea en el enunciado. Estas se analizan y se cuantifican en textos de naturaleza oral del Corpus Conversacional del Español de Canarias (CCEC). Los resultados indican que VSO y OVS son las más frecuentes en los medios de comunicación y en los textos conversacionales respectivamente. La primera conlleva un significado epistémico, que crea un estilo tendente a la objetividad, mientras que la variante OVS aporta un significado deóntico que es utilizado en los textos conversacionales para crear un estilo más subjetivo.

Palabras clave: variación, cognición, estilo, sujeto, objeto.

Cognitive salience and textual informativeness are tools revealed as very important in determinating the meaning created by syntactic variants in discourse. According to that we analyze three possible constructions of postverbal subject and object (VSO, VOS and OVS) in some oral texts from the Corpus Conversacional del Español de Canarias which comprises both mass media genres and spontaneous conversations. Results indicate that the most frequent variants are VSO and OVS being respectively used in mass media texts and spontaneous conversations.The former conveys an epistemic meaning helping to shape an objective style more suitable to be performed in mass media genres. Contrarily, the OVS variant showed up a deontic meaning which tends to create a more subjective style.

Key words: Variation, cognition, style, subject, object.

Este trabajo forma parte del desarrollo del proyecto de I+D “Desarrollo conceptual y análisis empírico de la variación sintáctica en español: los objetos verbales y las estructuras de pasiva” (FFI2013-41250-P) en el marco del Plan Estatal de Fomento de la Investigación Científica y Técnica de Excelencia del Ministerio de Economía y Competitividad. 


\section{INTRODUCCIÓN. LA VARIABILIDAD EN LA POSICIÓN DEL SUJETO PRONOMINAL}

La posición variable del sujeto pronominal en español (Yo canto/canto yo) es una propiedad muy conocida de la lengua española $y$, aunque ha sido un tema estudiado desde enfoques variacionistas tradicionales, permanece sin su completa resolución. El hecho de que el paradigma de los pronombres de sujeto pueda venir colocado antes o después del núcleo verbal es un tema que está relacionado con numerosos factores de distinta naturaleza; a simple vista podría parecer una mera cuestión de orden de palabras, pero la realidad es que este fenómeno conlleva variadas e importantes repercusiones de tipo significativo. Partimos de la base de que dos construcciones sintácticas diferentes llevan aparejadas un cambio en su significado (cf. Goldberg 1995: 67; Lakoff, 1987: 463) y que esta alteración, lejos de ser un problema para el sistema lingüístico, constituye un recurso para la creación de estilos comunicativos en el discurso y en la interacción (Serrano 2011:141-181; Serrano y Aijón Oliva 2011). El análisis de la variación sintáctica debe realizarse bajo la asunción de que la gramática es variable, que las variantes constituyen formas distintas de decir cosas distintas y de que esta variabilidad está sustentada en la cognición y debe ser explicada de acuerdo a ella (Aijón Oliva y Serrano 2013: 19, 26,143).

De hecho, en otros estudios se ha podido comprobar que la colocación preverbal o posverbal del sujeto pronominal puede explicarse a partir de los distintos grados de prominencia discursiva y de informatividad textual que adquiere cada una de estas variantes en el discurso (Serrano 2013a: 264; 2013b). La prominencia cognitiva es una operación de conceptualización cuya base reside en el proceso de atención, modelado en términos de activación mental; un elemento gramatical será prominente si está activado o es accesible en el discurso previo (v. Ariel 1990; Chafe 1986; Gundel y Fretheim 2009). De acuerdo con esto, es posible considerar que la colocación preverbal es más prominente o accesible que la posverbal, en tanto que es la información de la que se parte normalmente es temática o ya conocida. En el siguiente ejemplo, el pronombre nosotros presenta esas características y se formula al inicio de la intervención.

(1) Nosotros lo tenemos claro pero depende mucho de las plazas que haya para oposiciones $\backslash$ en realidad||parece que hay ochenta plazas\pendientes de eso $\varnothing$ estamos $\backslash$ (CCEC Med12<CanDi212>)

En cambio, cuando el sujeto viene colocado después del núcleo verbal, el pronombre de sujeto ocupa la posición sintáctica prototípica de los objetos, asume el contenido nocional y léxico del verbo y ello se resuelve en un mayor procesamiento cognitivo que se deposita en el referente del pronombre, propiedad fundamental de la norma textual de la informatividad (Beaugrande y Dressler 1997:47). La mayor informatividad del sujeto posverbal da lugar a variados significados basados en la focalización, novedad, imprevisibilidad o contraste. Estos pueden percibirse en los siguientes ejemplos.

(2) No se puede traer todo ese dinero del Estadolcreo yo

(CCEC Med12 <ElEnv2611>) 
(3) Pero se han pasado un pelín ha dicho usted len las medidas que se han tomado||sobre este asunto/ (CCEC Med12 <ElEnv2611 $>$ )

La colocación posverbal del sujeto representa, como cualquier otra variante sintáctica, una opción comunicativa para el hablante que se distribuye social y situacionalmente en distintos textos, como ya se ha comprobado (Serrano 2012 2013a).

La variación en la colocación del sujeto posee algunas propiedades sintácticas; en las cláusulas interrogativas y exhortativas la posición prototípica es la posverbal: “¿Qué opinas tú?”, “Imagínate tú”, pero el frecuente cambio a la posición preverbal del sujeto: “Tú qué opinas?, “Tú imagínate” aporta una mayor prominencia cognitiva al referente del mismo, asimilando la forma y el contenido de la cláusula a las declarativas, cuyo orden prototípico es SV (Aijón Oliva y Serrano 2012: 139). Por el contrario, cuando se altera el orden prototípico en estas cláusulas y se invierte (VS), se generan distintos significados basados en la informatividad que aporta al referente y a la cláusula la posición posverbal del sujeto: “Hoy cocinas tú” (Serrano y Aijón Oliva 2013).

\section{El sujeto posverbal y el objeto. Propiedades cognitivas.}

Para analizar y comprender de forma más precisa los significados que conlleva la posición posverbal del sujeto es necesario también observar la posición de otros elementos relevantes de la cláusula, como el objeto verbal directo con o sin preposición. Si el objeto se expresa, este normalmente ocupará la posición prototípica después del núcleo verbal (VO), si bien es muy frecuente que compita con el sujeto en su proximidad al mismo cuando aparece en posición posverbal (VSO o VOS). Asimismo, también es posible que el objeto aparezca en posición preverbal (OVS).

De acuerdo a la naturaleza morfosintáctica del objeto verbal (simple, compuesto y compuesto correferencial con un clítico), estas variantes pueden venir representadas del siguiente modo:

a) Cuando el objeto es simple:

(4a) Escribió María una carta (VSO)

(4b) Escribió una carta María (VOS)

(4c) Una carta escribió María (OVS)

b) Cuando el objeto es una proposición u oración compuesta:

(5a) Dígame usted cómo se llama (VSO)

(5b) Dígame cómo se llama usted (VOS)

(5c) Cómo se llama dígame usted (OVS)

c) Cuando el objeto aparece reproducido por un clítico verbal correferencial con el objeto, que pasa a ocupar la posición preverbal:

(6a) Le pregunté yo al oyente (VSO)

(6b) Le pregunté al oyente yo (VOS)

(6c) Al oyente le pregunté yo (OVS) 
Aunque los condicionantes gramaticales de estas tres posibilidades son muy variados y complejos, partiremos de un análisis cognitivo de las variantes VSO, VOS y OVS asignando distintos grados de prominencia-informatividad a cada una de ellas para posteriormente determinar los significados que generan en distintos textos y situaciones comunicativas.

La posición posverbal del objeto es la prototípica en español y es la que asume una mayor informatividad; el lexema verbal recae sobre el objeto, de modo que se produce un flujo o corriente de energía que se inicia en el sujeto (cuando este se coloca también prototípicamente en posición preverbal) y termina en el objeto paciente, siguiendo el modelo de evento canónico (v. Langacker 2000), claramente asociado a la transitividad, y por lo tanto, al objeto directo. Así, en el siguiente ejemplo la energía fluye desde el sujeto Juan hasta el objeto directo peras, pasando por el núcleo verbal come.

\section{(7) Juan come peras}

Sin embargo, cuando el sujeto se coloca tras el verbo, el objeto no deja de ser informativo, pero su percepción cognitiva y la informatividad será gradual y dependerá de la presencia del sujeto en esta misma posición. Distintos estudios han demostrado que cuando coexiste más de un elemento colocado tras el núcleo verbal, cada uno de ellos se ordena según distintos factores tales como la animacidad, el rol semántico de los constituyentes, el estatus de la información (cf. Siewierska 1995; Heidinger 2013: 160) o el peso sintáctico ${ }^{1}$ (Behaghel 1932: 6; Hawkins 2001). En español, el significado del orden se establece en buena medida por la naturaleza de la información, que viene relacionada con la tematización o focalización de los elementos que se sitúan respectivamente en primer o último lugar, lo cual se acompaña de un mayor o menor peso sintáctico respectivamente (Arnold et al., 2000: 34).

El marco teórico de este trabajo asume que cualquier variación en el plano sintáctico viene dada por la naturaleza intrínsecamente cognitiva de la gramática, así pues, el sujeto posverbal y el objeto adquirirán distintos grados de iconicidad cognitiva basados en la gradualidad de la prominencia cognitiva y de la informatividad textual, como se especifica a continuación.

a) Orden verbo-sujeto-objeto (VSO). En esta colocación el objeto sigue siendo informativo como cuando aparece inmediatamente detrás del verbo (VO), pero en ella se da la particularidad de que el sujeto puede presentarse como prominente al constituir un elemento ya conocido o accesible en el discurso. En los siguientes ejemplos se observa claramente la prominencia del pronombre usted (uno de los que con mayor frecuencia se expresa en posición posverbal) en cláusulas de tipo tiene usted, ha dicho usted, no me imagino yo, que presuponen que el referente ya ha sido previamente mencionado ${ }^{2}$.

Entendido como el número de elementos o constituyentes que conforman una función sintáctica. A mayor cantidad de ellos, mayor peso sintáctico.

2 Los verbos de lengua y conocimiento como decir, saber, imaginar, etc., son lexemas que muy frecuentemente se acompañan de la expresión de los sujetos pronominales (v. Enríquez 1984; Hurtado 2005), si bien lo hacen normalmente en posición preverbal. 
(8) Con lo que nos ha explicado sobre la situación de los enfermosltiene usted una labor ardua por delante $($ CCEC Med12 <ElEnv103 $>)$

(9) De los cinco millones de euros que se dedican a centros de acogida de la mujer ha dicho usted que no es necesaria tal partida $($ CCEC Med12 <ElEnv103>)

(10) No me imagino yo a un presidente de cabildo argumentando cualquier cosa (CCEC Med12<RoyCo2212>)

La posición del sujeto entre el verbo y el objeto (VSO) confiere al referente del sujeto cierta independencia con respecto al núcleo verbal ${ }^{3}$, de modo que su presencia parece servir a la función de mantener la referencia a lo largo del discurso y esto viene determinado porque el objeto presupone un aumento de la mayor informatividad y un descenso de la del sujeto. Hay que tener en cuenta la naturaleza del objeto en el orden VSO; si está constituido por una proposición compuesta, tendrá escasa o ninguna movilidad, de forma que lo más probable es que el objeto aparezca en posición final (cf. Heidinger 2013: 168)4.

(11) Se pregunta usted Carracedo $\backslash$ si cuando es en la costa es mejor que cuando aquel volcán en Islandia cerró el espacio europeolcon esa experiencia y otras que usted ha tenido... $\|($ CCEC Med12<ElEnv1310 $>)$

La prominencia del sujeto posverbal en la posición VSO se hace más visible en las cláusulas interrogativas tanto directas como indirectas, donde esta solución es la prototípica:

(12) Entonces ¿cómo ve usted los toros desde la barrera?`científicamente cómo se espera\ porque habrá sido muy distinto $\backslash$ (CCEC Med12<ElEnv1310>)

(13) Pilar/ ¿dónde está escuchando usted la radiol que parece una galería? (CCEC Med12<TertPe2612>)

(14) Díganos cómo va a rentabilizar usted esa mayoría absoluta)(CCEC Med12<ElEnv1010>

De hecho, en estas estructuras no es frecuente encontrar el orden VOS, precisamente porque en ellas el orden prototípico es VS, en el cual el sujeto ve reducida su agentividad a través de la colocación inmediatamente posterior al núcleo verbal ${ }^{5}$ (Aijón Oliva y Serrano 2012: 142; Cuartero Sánchez 2007). No obstante, en el corpus se ha obtenido algún ejemplo de interrogativas con orden VSO.

\footnotetext{
Como señala Padilla García (2001:247) en orden verbo-sujeto la acción y el sujeto se funden en la perspectiva del hablante y el sujeto parece esconderse tras el verbo.

4 De hecho, en un estudio sobre los órdenes sintácticos en polaco, Siewierska (1993:240) concluye que los constituyentes más cortos suelen aparecer antes que los largos.

5 No obstante, el cambio del VS hacia SV en las estructuras interrogativas es muy frecuente, y da lugar a distintos significados en el discurso (cf. Aijón Oliva y Serrano 2012).
} 
(15) ¿Tienen propuestas ustedes para reducir el fracaso escolar en el conjunto de Canarias? $($ CCEC Med12<ElEnv1310 $>$ )

b) Orden verbo-objeto-sujeto (VOS). El objeto alcanza su mayor nivel de informatividad cuando aparece junto al núcleo verbal (VO), pero el sujeto desplazado a la posición final después del objeto (VOS) resta informatividad a este y al asimilarse nocionalmente al objeto, se incrementa la del sujeto.

(16) Seguimos sintonizando lo que quieran ustedes en esta época de Navidad $\backslash$ (CCEC Med12<TertPe2811>)

(17) Así como en el mundo político se ha organizado un gran revuelolen el mundo cientifico se ha montado también un salpafuera $\backslash$ ha dicho usted $\mid$

(CCEC Med12<ETT2611>)

c) Orden objeto-verbo-sujeto (OVS). El desplazamiento del objeto a la posición preverbal hace que el sujeto quede en posición final y por lo tanto este adquiera mayor carga informativa de la que posee en las otras posiciones. En esta construcción el objeto pasa a ser prominente, su presencia en esta posición suele ser debida a que su referente es recuperable porque ha sido previamente activado, y es muy frecuente que venga representado por un clítico verbal. En (18) el objeto ejercicio se reproduce en las siguientes cláusulas por lo y en (19) el pronombre eso es correferencial con lo, y ambos constituyen el objeto directo de la estructura.

(18) Ya hice el ejercicio que me mandó la profesora\lo hice bien yolsi no lo llego a poner ahí estaría mal $\backslash(\mathrm{CCEC}$ Conv $<$ ElEn08 $>)$

(19) Eso es lo que estabas cantando tú por la mañana $($ CCEC Conv $<$ ElEn08>)

Asimismo, cuando en un mismo enunciado coaparecen dos objetos, uno de ellos puede aparecer expreso y el otro omitido correferenciado con un clítico verbal. En el siguiente ejemplo, el sujeto posverbal de la primera cláusula (usted) queda representado en la siguiente por el clítico le en la cláusula "Dios le guarde el consenso", mientras que el objeto consenso se repite en posición posverbal en ambas.

(20) Buenas noches $\backslash$ dice usted que ha habido consenso $\backslash$ pues Dios le guarde el consenso $\backslash$ esto ha sido idea suyalyo ya le he dicho que el consenso es el pleno $\|($ CCEC Conv $<$ ETT2611 $>$ )

De acuerdo con lo expuesto, podemos establecer una escala de prominencia y de informatividad para los sujetos y los objetos que integran cada variante. Cada una de estas variantes adquiere gradualmente la prominencia cognitiva y la informatividad textual tal y como queda representado en el siguiente esquema.

\section{Sujeto}

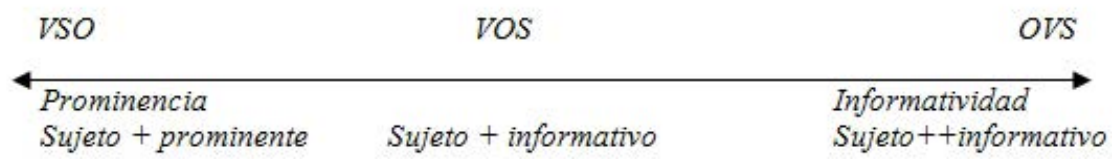

Gráfico 1. Escala de prominencia e informatividad del sujeto pronominal con respecto al objeto verbal 


\section{Objeto}

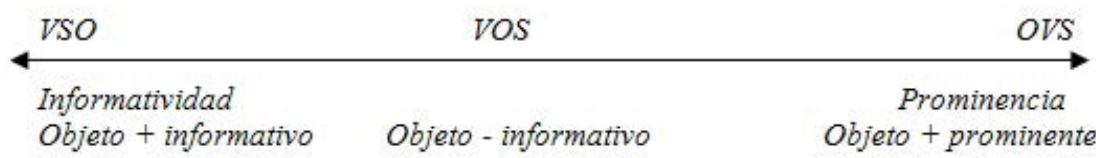

Gráfico 2. Escala de prominencia e informatividad del objeto verbal con respecto al sujeto pronominal

La gradualidad en la prominencia e informatividad que contiene cada una de estas variantes genera significados en la interacción comunicativa, que se distribuyen de distinta forma en los textos donde aparecen.

\section{Metodología y CORpus}

Estas tres variantes posicionales del sujeto-objeto serán analizadas en los textos del Corpus Conversacional de Canarias (CCEC), un corpus de español contemporáneo que contiene distintos géneros discursivos procedentes de los medios de comunicación, con un total de 171,258 palabras además de textos conversacionales espontáneos, sección que contiene 79,544 palabras. Para esta investigación se tendrán en cuenta únicamente los primeros, que cubren las principales modalidades discursivas de los géneros: a) magazines: programas de entretenimiento o variedades, en los que, gracias a su naturaleza diversa es posible encontrar distintas modalidades discursivas; b) informativos: programas dedicados estrictamente a la información donde a su vez aparecen secuencias de otros subtipos informativos: deportivos, meteorológicos, sociedad, entre otros; c) tertulias: reuniones de periodistas y políticos que tratan sobre un tema de actualidad; d) informativos-debates: programas dedicados a la información sobre temas políticos y de actualidad con la presencia de diversos hablantes que confrontan sus opiniones.

Con esta diversidad de géneros es posible obtener datos representativos de las variadas situaciones comunicativas donde tiene lugar la interacción entre los hablantes. No se trata de un corpus destinado al estudio de la variedad canaria, ni dicho estudio constituye el objetivo de este trabajo, no obstante, es obvio que los textos podrían de algún modo considerarse como representativos de esa variedad del español.

Se cuantificarán las variantes midiendo su frecuencia relativa en los distintos textos mediante el cálculo de los porcentajes, y cuando sea necesario, se aplicará la metodología de la variable absoluta, que relaciona la frecuencia de una forma por sí misma en relación con las características del entorno textual donde aparece y sus propiedades textuales. Este método normalmente cuantifica la frecuencia de una forma con respecto al número de palabras de un texto, dividiendo los casos obtenidos de la variante o variable por cada 10,000 palabras. No se trata solo de un simple procedimiento de cálculo, sino, además, una vía para explicar cómo se construye paralelamente la forma y el significado en un texto a partir de la cuantificación de su frecuencia en las situaciones comunicativas donde se utiliza y entre hablantes con distinta función social (Aijón Oliva y Serrano 2013: 64-67). 


\section{LA VARIACIÓN DE VSO, OVS Y VOS EN LOS GÉNEROS TEXTUALES}

Los significados de la posición posverbal pueden precisarse a partir de la observación de la colocación del objeto en distintos géneros textuales. En otras investigaciones se ha determinado que la frecuencia de la posición posverbal en español es muy escasa (Brown y Rivas 2011; Enríquez 1984; Meyer-Hermann 1990; López Meirama 1997; Morales 1999; Orozco y Guy 2004; Otheguy y Zentella 2007). El corpus que analizamos sigue esta tendencia y los sujetos en posición posverbal constituyen solo un $4.5 \%$ del total. La frecuencia en los textos conversacionales casi dobla a la de los medios de comunicación (Tabla I).

Tabla I. Porcentajes de sujeto posverbal en los textos del CCEC

\begin{tabular}{|l|c|c|c|}
\hline & Sujeto posverbal & Total de sujetos & $\%$ \\
\hline Textos conversacionales & 101 & 1474 & $6,9 \%$ \\
\hline $\begin{array}{l}\text { Textos de los medios de } \\
\text { comunicación }\end{array}$ & 115 & 3311 & $3,5 \%$ \\
\hline Total & 216 & 4785 & $4,5 \%$ \\
\hline
\end{tabular}

Por otra parte, como se observa en la tabla II, los sujetos en posición posverbal coaparecen con objetos en un 55\% de los casos en los dos tipos de texto, si bien en aquellos de los medios de comunicación la frecuencia es superior $(62,6 \%)$, lo cual puede venir determinado por la variada naturaleza de estos géneros, donde, como se explicará después, podría ser más necesaria la expresión del objeto verbal (Tabla II).

Tabla II. Porcentajes de coaparición del sujeto posverbal con objetos sintácticos

\begin{tabular}{|l|c|c|}
\hline & Presencia de objeto & $\%$ \\
\hline Textos conversacionales & $47 / 101$ & $6,9 \%$ \\
\hline $\begin{array}{l}\text { Textos de los medios de } \\
\text { comunicación }\end{array}$ & $72 / 115$ & 62,6 \\
\hline Total & $119 / 216$ & 55 \\
\hline
\end{tabular}

Con respecto a las variantes de la colocación del sujeto y del objeto, la distribución es dispar; es posible observar que el orden VSO es claramente predominante en los textos de los medios de comunicación, mientras que VOS y, sobre todo OVS, lo son en los conversacionales (Tabla III).

Tabla III. Porcentajes de las variantes VSO, VOS y OVS en los textos del CCEC

\begin{tabular}{|l|c|c|c|c|}
\hline & VSO & VSO & OVS & Total \\
\hline Textos conversacionales & Total & $8(17 \%)$ & $22(46.9 \%)$ & 47 \\
\hline $\begin{array}{l}\text { Textos de los medios de } \\
\text { comunicación }\end{array}$ & $58(80,6 \%)$ & $4(5,5 \%)$ & $10(13.9 \%)$ & 72 \\
\hline Total & $75(63 \%)$ & $12(10.1 \%)$ & $32(26.9 \%)$ & 119 \\
\hline
\end{tabular}

Esto apunta a una primera conclusión; no solo la presencia y expresión del objeto verbal, sino también el orden prototípico que sitúa al objeto en la posición final de la cláusula es más frecuente en los textos con una mayor tendencia a la planificación o con menor espontaneidad, como lo son, en principio (aunque no categóricamente) 
los de los medios de comunicación. El objeto en las posiciones preverbal (OVS) y, en menor medida, intermedia (VOS), son más frecuentes en los textos de naturaleza básicamente espontánea, como los conversacionales.

No obstante, estas apreciaciones deberán ser matizadas con vistas a determinar el estilo comunicativo creado con estas variantes y ello tiene que estar lejos de percepciones intuitivas, vagas e imprecisas que califican al estilo como de coloquial, oral, (in)formal o vulgar, entre otras denominaciones. Partimos de una idea más dinámica de la clásica dicotomía coloquial-formal paralelamente asociada a las dimensiones oral-escrita de la lengua en la que se ordenan los estilos lingüísticos.

La base cognitiva que sustenta la variación especifica qué tipo de significado se crea con ellas y es en definitiva lo que permite delinear la naturaleza del estilo comunicativo (Serrano 2011: 159-173). Para ello, será necesario analizar más detalladamente las frecuencias de las variantes en los distintos géneros textuales del corpus.

Dada la escasa representatividad en porcentajes de la variante VOS, a partir de aquí el análisis se centrará en las más frecuentes, esto es, VSO en los medios de comunicación y OVS en los textos conversacionales. Los cálculos absolutos de cada una de ellas miden su densidad en un tipo de texto, lo cual demuestra que la variación y el texto o discurso se construyen mutuamente.

\subsection{Frecuencias y significado de VSO en los medios de comunicación}

El cómputo de la distribución de esta variante por cada 10,000 palabras arroja los siguientes resultados (Tabla IV).

Tabla IV. Frecuencias absolutas de la variante VSO en los géneros textuales de los medios de comunicación del CCEC

\begin{tabular}{|l|c|c|}
\hline & Casos & Frecuencia \\
\hline Informativos & 10 & 1,8 \\
\hline (55, 474 palabras) & 14 & 3,3 \\
\hline (41, 753 palabras) & 3 & 2,3 \\
\hline $\begin{array}{l}\text { Magacines } \\
(61,346 \text { palabras })\end{array}$ & 31 & 5 \\
\hline Total & 58 & 3,3 \\
\hline
\end{tabular}

Son los magacines y los informativos-debate los géneros donde el orden VSO es más frecuente, y ello es debido a que, si bien son textos con características diferentes, comparten el objetivo de tratar cierta variedad de temas con distintos objetivos: el entretenimiento y la información respectivamente. Mientras los magacines son programas de variedades sin una orientación comunicativa concreta, los informativosdebate son géneros que combinan la información en la forma tradicional con el debate de los temas que la conforman. En ambos existe una tendencia a la interacción, si bien más en los primeros que en los segundos, con lo cual es esperable que una de las formas empleadas usuales sea la del uso de la cláusula interrogativa (tanto directas como indirectas), que tiende a utilizar la estructura VSO, como se observa en los siguientes ejemplos. 
(21) [Magacine]

¿Va a comprar usted a este tipo de clientes?/lo que estamos produciendo no se está vendiendo al precio que tiene que venderse $\backslash(\operatorname{Med} 12<\mathrm{R} 72010>)$

(22) [Informativo-debate]

Se abre la seguridad de Los Roquillos ¿Es usted partidario de esa decisión?/ $($ Med12<ETT2611 $>$ )

\section{(23) [Magacine]}

Antes de decirles adiós y agradecerles que hayan compartido con nosotros su tiempo sintonizando/ les pediríamos que nos dijeran cómo dibujarían ustedes este 2011 (CCEC Med12<TePe2811)

La mayor prominencia del referente del sujeto y la mayor informatividad del objeto que conlleva este orden parece apropiado para este tipo de enunciados. La prominencia del primero resulta de su accesibilidad en el discurso, que proviene de la continuidad de la interacción o diálogo; en estos géneros, el interrogado (sea la primera o segunda persona) está presente físicamente o no. En cuanto al objeto, que aparezca expreso y al final de la cláusula concuerda también con el tipo de interacción, puesto que aporta la informatividad apropiada para focalizar el contenido de la pregunta.

La prominencia del sujeto y la informatividad del objeto verbal pospuesto ${ }^{6}$ en el orden VSO aportan al enunciado un significado basado en la modalidad epistémica. Esta modalidad es una concepción discursiva amplia y no exenta de variadas matizaciones (v. Nuyts 2001:25-28; Weatherson y Egan 2011: 7-11) sin embargo, el sentido que se toma en este trabajo de significado epistémico proviene de la capacidad que muestra el hablante de exponer el contenido proposicional conforme a evidencias de tipo personal, basadas en su propia capacidad agentiva o bien en fuentes externas de información (Nuyts 2001: 26). El hablante aporta validez a los hechos o constatación de su veracidad; en definitiva, adopta una perspectiva de responsabilidad evidencial sobre lo que está diciendo y, desde el punto de vista sintáctico, estas evidencias recaen nocionalmente sobre el objeto. Esto lo hace especialmente apropiado para aquellas interacciones donde se pretende potenciar la objetividad de los hechos narrados o los contenidos expresados, como los informativos debate y, en menor medida, los magacines, donde esto estará determinado por la variedad de temas tratados en ellos.

El significado epistémico de VSO puede observarse tanto en las estructuras interrogativas como en las declarativas.

a) El orden VSO en las estructuras interrogativas. El objeto constituye el foco informativo de la cláusula (como cabría esperar en un esquema prototípico de la interrogación) y en él recae la evidencia que sostiene el significado epistémico. Se ha demostrado que, aparte de la modalidad interrogativa que les corresponde, estas estructuras constituyen entornos sintácticos donde puede apreciarse la modalidad epistémica (Frajzingyer 1995:476; Squartini 2010:110, 112; Pietrandrea 2005: 131) básicamente porque en ellas se plantean conjeturas o dudas, que pueden estar fundamentadas en hechos concretos establecidos por el hablante y/o basados en su

En otro trabajo se ha demostrado que, en virtud de la informatividad, la colocación posverbal del sujeto pronominal promueve un significado epistémico-evidencial (Serrano 2013a: 275). 
responsabilidad $^{7}$ que necesitarían confirmación por parte del oyente. Esto puede observarse en los siguientes ejemplos de estructuras interrogativas totales.

(24) [Informativo-debate]

¿No cree usted que ha sido un tanto irresponsable con sus declaraciones desde el primer momento?/ (Med12<ETT2611>)

(25) [Magacine]

Lo que estamos produciendo no se está vendiendo al precio que tiene que venderselpero la gente lo sigue comprando ¿Va a vender usted a este tipo de cliente?/ (Med12<R72010>)

b) El orden VSO en las cláusulas declarativas. En los siguientes ejemplos de cláusulas declarativas puede comprobarse que el orden VSO aporta un significado epistémico, ya que se presentan como hechos o estados de cosas basados en evidencias; así en (26) se señala: “Tiene usted una labor ardua por delante” y a continuación se da la explicación: "porque tenemos..."

(26) [Informativo-debate]

Tiene usted una labor ardua por delante $\backslash$ porque tenemos un punto más de pobrezaly tenemos que apelar a la voluntad de los trabajadores $\backslash(\operatorname{Med} 12<$ ElEnv1013 $>)$

En (27), la cláusula "No me imagino yo a un presidente del Cabildo", viene refrendada por una evidencia personal: "yo creo que lo que ha dicho se va a debatir..."

(27) [Magacine]

No me imagino yo a un presidente del Cabildo argumentando cualquier cosalyo creo que lo que ha dicho se va a debatir el próximo viernes $\backslash(\operatorname{Med} 12<$ RoyCo222 $>)$

La estructura "Ha dicho usted que no es necesaria tal partida..." en (28) adquiere un significado epistémico a partir de la evidencia que proporciona la primera proposición: "De los cinco millones de euros que se dedican..."

(28) [Informativo-debate]

De los cinco millones de euros que se dedican a centros de acogida de la mujer ha dicho usted que no es necesaria tal partida (Med12<ElEnv103>)

Por tanto, es posible determinar que la secuencia de órdenes VSO proporciona a los enunciados un significado basado en la epistemicidad a partir de la menor prominencia cognitiva del sujeto y la mayor informatividad del objeto.

\subsection{El orden OVS en los textos conversacionales}

El orden OVS supone una alteración de las posiciones prototípicas de ambos elementos que implica un intercambio en la percepción cognitiva; el objeto ocupa

\footnotetext{
Es posible establecer un subdominio modal correspondiente a la conjetura, que permitiría una definición más concreta del significado epistémico en las estructuras interrogativas (Squartini 2010:112). Según Verstraete (2001: 1523) este significado establece una posición sobre el contenido proposicional y este puede producirse tanto en las estructuras declarativas como en las interrogativas; el hablante puede tomar la responsabilidad de ese contenido en su turno de palabra y cederlo al oyente en la suya en las cláusulas interrogativas. En estas, el origen de la evaluación del contenido no está en el oyente, sino en el interlocutor que va a responder la pregunta.
} 
la posición del sujeto y este la del objeto. Dicho cambio en las posiciones conlleva aparejado un significado opuesto a la epistemicidad; si el objeto se transforma en prominente o accesible, la carga informativa ya no viene asumida por él, sino por el sujeto.

El intercambio de estas funciones da lugar a un significado deóntico basado en la evaluación moral de la aceptabilidad, deseo o necesidad de un estado de cosas que incluye nociones como exhortación, obligación o el permiso exigido por un agente externo para el cumplimiento del contenido (Kratzer 1978: 111; Nuyts 2001:25; Palmer 2001: 9; Von Wright 1951: 1-2). La modalidad deóntica vincula un agente con un estado de cosas, esto es, puede implicar al sujeto como responsable o depositario del contenido del lexema verbal. Esto puede observarse en la estructura OVS fundamentalmente a partir de la informatividad que adquiere el sujeto, que ocupa la posición sintáctica prototípica del objeto, que se convierte en el foco de la predicación.

De hecho, el significado que adquiere el orden OVS es asimilable al exhortativoimperativo propio de las estructuras interrogativas parciales y de las imperativas, donde el objeto suele situarse al principio de la estructura y el sujeto al final de la misma ${ }^{8}$ :

(29) ¿Qué quieres que haga yo?

(30) La ensalada, te la comes tú

Es posible observar que las estructuras con orden OVS exhiben cierto significado de expresión de deseo o exhortativo, que se realiza de forma más apta a través de la segunda persona del paradigma pronominal.

(31) Mejor lo hace usted en el taller $\backslash$ porque aquí vamos a hacer una chapuza $\backslash$ (CCEC $<$ ElEn08>)

No obstante, es posible encontrarlas también con la primera persona, como en el siguiente ejemplo, donde el propio hablante expresa la necesidad u obligación de que el contenido se cumpla, como en el siguiente ejemplo.

(32) [Conversación espontánea]

A mí me sugirió que la demanda la tengo que presentar yol porque soy delegada sindical y yo lo consulté abajo y me dicen que no $\backslash$ que no tiene sentido $($ CCEC $<$ ElEn08>)

(33) [Conversación espontánea]

Hay que tener ficha financiera como lo dije yo y como lo dijo el presidentell(CCEC $<$ ElEn08>)

En cualquier caso, el rasgo común de estas construcciones es que la colocación del objeto preverbal y del sujeto posverbal en conjunto procura un significado deóntico, basado en el deseo o compromiso de que las acciones o contenidos se lleven a cabo, que contrasta con la epistemicidad de VSO. En (34) el hablante expresa su intención de realizar la acción situando el sujeto yo en posición posverbal que asume la carga informativa de la cláusula, mientras que el objeto (esto, lo) es el elemento del que se parte para realizar la predicación, que es accesible o está activado ya en el discurso previo.

No obstante, en las estructuras imperativas es posible que el objeto aparezca pospuesto en forma de clítico: "La ensalada, cómetela tú". 
(34) [Conversación espontánea]

Esto lo desarmo yolo meto en un furgón y me lo llevo pa Cádiz\ (CCEC $<$ ElEn08>)

En este otro, el significado deóntico surge mediante la afectación que sufre el referente del sujeto yo en posición posverbal. Por su parte el objeto omitido (la Navidad) correferenciado mediante un clítico (la) resulta ser información conocida que no desea ser focalizada en el enunciado.

(35) [Conversación espontánea]

El año pasado estaba mala, casi no podía ni comer ni nada||pero no fui porque no quería yo ir ||| y un año la (la Navidad) pasé yo en la Residencia\ (CCEC $<$ ElEn08>

No es casual que en casi todos los ejemplos encontrados el objeto venga reproducido por un clítico verbal, lo cual confirma la idea de que el objeto, en las construcciones OVS es cognitivamente prominente, y que esto conlleva un claro incremento de la carga informativa del sujeto.

(36) [Conversación espontánea]

Y encimalesto lo pagaste tú a tocateja \sin préstamos ni nada|||

$($ CCEC $<$ ElEn08>)

\section{HACIA UNA DELIMITACIÓN DEL ESTILO COMUNICATIVO}

Las características cognitivas de cada una de las variantes, basadas en la gradualidad de la prominencia cognitiva y la informatividad textual promueven dos significados basados en las modalidades deóntica y epistémica respetivamente. Que cada una de estas variantes obtenga frecuencias distintas en cada tipo de género textual indica que la variación sintáctica es un elemento importante en la construcción de los estilos comunicativos de los géneros textuales.

Desde un punto de vista cognitivo como el adoptado en este trabajo, los estilos se inscriben en un continuum que va de la objetividad hacia la subjetividad (v. Aijón Oliva y Serrano 2013:145).

\section{Objetividad}

Subjetividad

Gráfico 3. Continuum estilístico de la objetividad-subjetividad

Así pues, el orden VSO es más frecuente en los textos de los medios de comunicación y, más concretamente, en aquellos más proclives a la interacción con otros hablantes, presentes o no en la emisión, como los magacines y los informativosdebate. La presencia del objeto y su posición posverbal completan la predicación del verbo, de forma que el significado epistémico surge en forma de enunciados interrogativos o de estructuras declarativas a partir de la inquisición de información o de la formulación de evidencias basadas en datos reales, o que se presentan como de conocimiento compartido o general. 
Por su parte, la modalidad epistémica podrá ser más o menos objetiva en función del punto de vista que plantee el hablante sobre los hechos descritos (cf. GonzálezVázquez 1998:352). A partir de las características cognitivas descritas del orden VSO que dan lugar al significado epistémico, es posible establecer que esta contribuye a la creación de un estilo comunicativo basado en la objetividad. Este significado es acorde con el tipo de texto donde más frecuentemente aparece la estructura VSO (magacines e informativos-debate y magacines) ya que, además de la transmisión de información, en ellos predomina respectivamente el entretenimiento y la interacción entre hablantes.

Al contrario que la subjetividad, la objetividad se organiza a partir de la estructura lingüística y es independiente de la participación o percepción del hablante sobre los hechos descritos (Farrar y Jones 2002:6), e incluso de cuestiones culturales o sociales (Gaukroger 2012:42). La construcción de la objetividad está basada en la focalización lingüística sobre los objetos, mientras que la subjetividad lo hace sobre los sujetos (Langacker 1994: 15-16). De esta forma, es posible establecer que la presencia de elementos que contribuyen a la creación de estilos más objetivos no está necesariamente vinculada a los textos informativos, sino también a otras funciones del discurso, como la interacción o la dialogicidad.

Por su parte, la modalidad deóntica, aunque puede ser objetiva, presenta cierta tendencia a la subjetividad; el hablante no relata los hechos como un observador neutral, sino que expresa sus creencias y actitudes (Lyons 1981:237; Vázquez-Laslop 1999:7). Esto no significa que el significado deóntico deba asociarse siempre a la subjetividad; la naturaleza cognitiva del orden OVS, que implica, como se ha visto, una alteración de la posición prototípica del objeto y del sujeto, produce distintos valores significativos basados en el correspondiente intercambio de la gradualidad de la prominencia cognitiva y de la informatividad: el sujeto es más informativo y el objeto se transforma en prominente. El significado deóntico que proporciona la variante OVS está relacionado con un compromiso o posición del hablante en el contenido a partir de la relación sintáctico-discursiva establecida entre ambos elementos; la subjetividad que proporciona el sujeto expreso ${ }^{9}$ como responsable o participante del contenido, estará matizada por la prominencia que aporta el objeto (generalmente clítico verbal) debido a que su referencia ya ha sido previamente mencionada en el discurso.

No cabe afirmar, por tanto, que el significado deóntico sea por sí mismo inductor de subjetividad, aunque en el continuum estilístico propuesto este implica cierta tendencia a ella. Su elevada frecuencia en los textos conversacionales tampoco debe asociarse completamente a la tradicional subjetividad atribuida a estos textos ${ }^{10}$. El hecho de que la mayor parte de los ejemplos de OVS estén construidos con un clítico en posición preverbal y que este sea cognitivamente prominente es algo habitual en las conversaciones espontáneas, en las que predomina la continuidad discursiva (véanse los ejemplos (31)-(36)) cuyo mantenimiento suele hacerse mediante tales elementos prominentes.

De hecho, en otros trabajos se ha demostrado que el sujeto expreso en posición posverbal contribuyen a la creación de un estilo tendente a la objetividad (Serrano 2012).

10 Desde una perspectiva cognitiva, se ha demostrado, por ejemplo, que la presencia de la primera persona 'yo' no es más frecuente en los textos espontáneos que en los de los medios de comunicación (Serrano 2014b). 
Por otra parte, tanto en los textos de los medios de comunicación como en los conversacionales es interesante también tener en cuenta el rol que desempeña el hablante en ellos, ya sea su adscripción socioprofesional (políticos, periodistas, hablantes particulares y profesionales) o bien la función comunicativa que desempeña (transaccional o interpersonal) (v. Serrano 2013a: 266). No obstante, la escasez de ejemplos de sujetos pronominales en posición posverbal no permitiría obtener conclusiones demasiado relevantes.

\section{Conclusiones}

La variación sintáctica es un elemento de especial importancia para la comprensión de los objetivos comunicativos de los géneros y textos donde aparece. En concreto, a partir de trabajos previos (véase un resumen en Serrano 2014a), se ha podido demostrar la creación de estilos comunicativos a partir del estudio de la variación de los sujetos pronominales en español.

La combinación variable del sujeto posverbal con el objeto, en sus tres posibilidades VSO, VOS y OVS, adquiere distintos grados de prominencia cognitiva e informatividad textual. A partir de ellas, las dos variantes más frecuentes (VSO y OVS) generan dos tipos de significado discursivo-pragmático fundamentado en las modalidades epistémica y deóntica. Su distribución en frecuencias absolutas en los diferentes géneros textuales (conversacionales y de los medios de comunicación) indica que dicho significado ayuda a definir la naturaleza textual de cada uno de ellos mediante el estilo comunicativo creado. El valor epistémico de VSO se origina fundamentalmente por la carga informativa del objeto verbal y la prominencia del sujeto y es más frecuente en las estructuras interrogativas y declarativas de los magacines e informativos-debate, todo lo cual indica que en ellos existe una tendencia estilística hacia la objetividad.

Por el contrario, la inversión en las posiciones del objeto y del sujeto con respecto al verbo lleva aparejado un cambio en las propiedades cognitivas adquiridas por cada elemento del que surge el significado deóntico. Este resulta más apto en aquellas circunstancias comunicativas donde predomina la espontaneidad y la continuidad discursiva, lo que da lugar a un estilo comunicativo tendente a la subjetividad.

\section{OBRAS CITADAS}

Aijón Oliva, Miguel A. y Serrano, María José. 2012b. "La posición del sujeto pronominal en las cláusulas no declarativas”. Onomázein 26: 131-164.

Aijón Oliva, Miguel A. y Serrano, María José. 2013. Style in Syntax. Investigating Variation in Spanish Pronoun Subjects. Bern: Peter Lang.

Ariel, Mira. 1990. Accessing Noun Phrase Antecedents. Londres: Routledge.

Arnold, Jennifer, Wasow, Thomas, Losongco, Anthony y Ginstrom, Ryan. 2000. "Heaviness vs. newness: The effects of structural complexity and discourse status on constituent ordering”. Language 76: 28-55.

Beaugrande, Robert y Dressler, Wolfgang U. 1997. Introducción a la lingüística del texto. Barcelona: Ariel.

Behaghel, Otto. 1932. Deutsche Syntax: Eine geschichtliche Darstellung: Band IV: Wortstellung. Periodenbau. Heidelberg: Carl Winters Universitätsbuchhandlung. 
Bellosta von Colbe, Valeriano. 2005. "Variación sintáctica en el "Role and Reference Grammar": La posición de los complementos en las oraciones ditransitivas. En Gabriele Knauer y Valeriano von Bellosta Colbe (eds.), Variación sintáctica en español: Un reto para las teorías de la sintaxis. Tübingen: Niemeyer, 97-116.

Bolinger, Dwight. 1954. "English prosodic stress and Spanish sentence order”. Hispania 37: 152-156.

Brown, Esther L. y Rivas, Javier. 2011. "Subject-Verb word order in Spanish interrogatives: A quantitative analysis of Puerto Rican Spanish". Spanish in Context 8: 23-49.

Chafe, Wallace L. 1986. "Evidentiality in English conversation and academic writing". En Wallace L. Chafe y Johanna Nichols (eds.) Evidentiality: The Linguistic Coding of Epistemology. Norwood: Ablex, 261-272.

Cuartero Sánchez, Juan Manuel. 2007. "Sobre la naturaleza y el alcance de los factores que influyen en el orden de palabras (con referencia a las oraciones declarativas en español actual)”. Lingüística Española Actual 29: 101-118.

Enríquez, Emilia. 1984. El pronombre personal sujeto en la lengua española hablada en Madrid. Madrid: CSIC.

Farrar, Kimberley y Jones, Mari C. 2002. "Introduction”. En Mari C. Jones y Edith Esch (eds.) Language Change: The Interplay of Internal, External and Extra-linguistic Factors. Berlín: Mouton de Gruyter, 1-18.

Frajzyngier, Zygmunt. 1995. "A functional theory of complementizers". En Joan Bybee y Suzanne Fleischmann (eds.), Modality in Grammar and Discourse. Amsterdam: Benjamins, 473-502.

Gaukroger, Stephen. 2012. Objectivity: A Very Short Introduction. Oxford: Oxford University Press.

Goldberg, Adele .E. 1995. Constructions. A Construction-Grammar Approach to Argument Structure. Chicago: The University of Chicago Press.

González-Vázquez, Mercedes. 1998. "El ámbito de actuación de los contenidos modales: los niveles de la modalidad". En Gilles Luquet (dir.) Travaux de Linguistique Hispanique. París: Presses Sorbonne Nouvelle, 347-355.

Gundel, Jeanette K. y Fretheim, Thorston .2009. "Information structure". En Frank Brisard et al. (eds.) Grammar, Meaning and Pragmatics, Amsterdam: Benjamins, 146-160.

Gutiérrez-Bravo, Rodrigo. 2007. Prominence scales and unmarked word order in Spanish. Natural Language \& Linguistic Theory 25: 235-271.

Hawkins, John A. 2001. “Why are categories adjacent?” Journal of Linguistics 37: 1-34.

Heidinger, Steffen. 2013. "Information focus, syntactic weigth and postverbal constituent order in Spanish”. Borealis. An International Journal of Hispanic Linguistics 2. 2: 159-190.

Hurtado, Luz Marcela. 2005. "Condicionamientos sintáctico-semánticos de la expresión del sujeto en el español colombiano”. Hispania 88. 2: 335-348.

Kratzer, Angelika. 1978. Semantik der rede. Scriptor: Königstein.

Lakoff, George. 1987. Women, Fire, and Dangerous Things: What Categories Reveal about the Mind. Chicago: University Press.

Langacker, Ronald. W. 1994. "The limits of continuity: Discreteness in cognitive semantics". En Catherine Fuch y Bernard Victorri (eds.) Continuity in Linguistic Semantics. Amsterdam: Benjamins, 9-20.

Langacker, Ronald W. 2000. "Estructura de la cláusula en la gramática cognoscitiva". En Ricardo Maldonado (ed.) Estudios cognoscitivos del español. Volumen monográfico de la Revista Española de Lingüística Aplicada, 19-65.

López Meirama, Belén. 1997. La posición del sujeto en la cláusula monoactancial en español. Santiago de Compostela: Universidad de Santiago de Compostela.

Lyons, John. 1981. Language, Meaning and Context. Londres: Fontana.

Meyer-Hermann, Reinhard. 1990. "Sobre algunas condiciones pragmáticas de la posición del sujeto en español”. Estudios de Lingüística de la Universidad de Alicante 6: 73-88. 
Morales, Amparo. 1999. "Anteposición del sujeto en el español del Caribe". En Luis Ortiz (ed.) El Caribe hispánico: perspectivas lingüísticas actuales. Madrid: Iberoamericana, 77-98.

Nuyts, Jan. 2001. Epistemic Modality, Language and Conceptualization. Amsterdam: Benjamins.

Ocampo, Francisco. 1990. "The pragmatics of word order in constructions with a verb and a subject”. Hispanic Linguistics 4: 87-128.

Orozco, Rafael y Guy, Gregory R. 2008. "El uso variable de los pronombres sujetos: ¿qué pasa en la costa caribe colombiana?" En Maurice Westmoreland y Juan Antonio Thomas (eds.) Selected Proceedings of the Fourth Workshop on Spanish Sociolinguistics. Massachusetts: Somerville, 70-80.

Otheguy, Ricardo y Zentella, Ana Celia .2007. "Apuntes preliminares sobre el contacto lingüístico y dialectal en el uso pronominal del español en Nueva York". En Kim Potowski y Richard Cameron (eds.) Spanish in Contact: Policy, Social and Linguistic Inquiries. Amsterdam: Benjamins, 275-295.

Padilla García, Xosé A. 2001. El orden de palabras en el español coloquial. Tesis doctoral, Valencia: Universidad de Valencia.

Palmer, Richard F. 2001. Mood and Modality. Cambridge: Cambridge University Press.

Pietrandrea, Paola. 2005. Epistemic Modality. Functional Properties and the Italian System. Amsterdam: Benjamins

Serrano, María José. 2011. Sociolingüística. Barcelona: Ediciones del Serbal.

Serrano, María José. 2012. "El sujeto pronominal usted/ustedes y su posición: variación y creación de estilos comunicativos”. Spanish in Context 9.1:109-131.

Serrano, María José. 2013a. "Variación sociosituacional de la colocación del sujeto pronominal en textos conversacionales". Spanish in Context 10. 2: 261-283.

Serrano, María José. 2013b. "De la cognición al discurso: el efecto de la prominencia cognitiva y la informatividad textual en el estudio de la variación de los sujetos pronominales". Estudios de Lingüística de la Universidad de Alicante 27: 275-299.

Serrano, María José. 2014a. "La variación sintáctica". En Javier Gutiérrez-Rexach (ed.) Enciclopedia de Lingüística Hispánica. Londres: Routledge (en prensa).

Serrano, María José. 2014b. "El sujeto y la subjetividad: variación del pronombre yo en géneros textuales del español de Canarias”. Signos. Estudios de Lingüística 47, 85: 321-343.

Serrano, María José y Miguel A. Aijón Oliva .2011. "Syntactic variation and communicative style”. Language Sciences 33: 138-153.

Serrano, María José y Miguel A. Aijón Oliva .2013. "El sujeto posverbal: función pragmática y cognición en las cláusulas declarativas”. Neuphilologische Mitteilungen 79: 309-331.

Siewierska, Anna. 1993. "Syntactic weight vs information structure and word order variation in Polish”. Journal of Linguistics 29: 233-265.

Siewierska, Anna. 1995. "On the interplay of factors in the determination of word order". En Joachim Jacobs, Arnim von Stechow, Wolfgang Sternefeld y Theo Vennemann (eds.) Syntax: An International Handbook of Contemporary Research / Ein Internationales Handbuch zeitgenössischer Forschung. Berlín/ New York, Walter de Gruyter, 826846.

Squartini, Mario .2010. "Where mood, modality and illocution meet: the morphosyntax of Romance conjectures". En Martin G. Becker y Eva María Remberger (eds.) Modality and Mood in Romance. Berlín: Walter de Gruyter, 109-129.

Vázquez Laslop, María Eugenia. 1999. "Modalidad deóntica objetiva y subjetiva". Nueva Revista de Filología Hispánica 47. 1: 1-32.

Verstraete, Jean Christophe. 2001. "Subjective and objective modality: Interpersonal and ideational functions in the English modal auxiliary system". Journal of Pragmatics 33: $1505-1528$. 
Von Wright, Georg Henrik. 1951. An Essay in Modal Logic. Amsterdam: North Holland. Weatherson, Brian y Andy Egan. 2011. "Introduction: Epistemic modals and epistemic modality". En Brian Weatherson y Andy Egan (eds.) Epistemic Modality. Oxford: University Press, 1-18. 